\title{
Urine Neutrophil Gelatinase-Associated Lipocalin (UNGAL) as a Marker for Acute Kidney Injury in Kidney Surgery Patients
}

\author{
Preston C. Sprenkle, James Wren, Alexandra C. Maschino, Andrew Feifer, Nicholas Power, \\ Tarek Ghoneim, Itay Sternberg, Martin Fleisher, and Paul Russo \\ Urology Service, Department of Surgery (PCS, JW, AF, NP, PR), Department of Epidemiology \\ and Biostatistics (AM), Sloan-Kettering Institute (TG), Clinical Chemistry Service, Department of \\ Laboratory Science (MF), Memorial Sloan-Kettering Cancer Center, New York, New York, USA
}

\begin{abstract}
Purpose-Evaluate uNGAL as a marker for AKI in patients undergoing PN to identify the preoperative clinical features and surgical factors during PN that are associated with renal injury as measured by increased uNGAL levels compared to controls.
\end{abstract}

Methods-Using RN and thoracic surgery patients as control groups, we prospectively collected and analyzed urine and serum of PN, RN, and thoracic surgery patients between April 2010 and April 2012. Urine was collected preoperatively and at multiple time points postoperatively. Differences in uNGAL levels between the 3 surgical groups were analyzed using a GEE model. The PN group was subdivided based on preoperative eGFR $<60$ or $260 \mathrm{ml} / \mathrm{min} / 1.73 \mathrm{~m} 2$.

Results-Of 162 patients included in the final analysis, $>65 \%$ had CVD, and median eGFR was $>60 \mathrm{ml} / \mathrm{min} / 1.73 \mathrm{~m} 2$ for all groups $(\mathrm{RN}=61, \mathrm{PN}=78$, thoracic surgery=84.5 $\mathrm{ml} / \mathrm{min} / 1.73 \mathrm{~m} 2)$. Preoperatively, a 10-unit increase in eGFR was associated with a 4- unit decrease in uNGAL in the PN group. Postoperatively, uNGAL levels in the PN group were not higher than thoracic surgery or RN control groups, and did not correlate with duration of ischemia. PN patients with preoperative eGFR <60 developed higher uNGAL levels postoperatively compared to those with a higher preoperative eGFR.

Conclusion-uNGAL does not appear to be a useful marker for detection of renal injury in healthy PN patients. However, patients with poorer preoperative renal function have higher baseline uNGAL levels and appear more susceptible to AKI as detected by uNGAL levels and AKIN criteria compared to those with normal eGFR.

\section{Keywords}

NGAL protein; human; acute kidney injury; biological markers; nephrectomy; surgery

Corresponding Author: Preston Sprenkle, MD Department of Urology Yale University School of Medicine PO Box 208058 New Haven, CT 06520-8058 Tel: 203-785-2815.

No financial disclosures 


\section{INTRODUCTION}

PN is associated with less renal function loss compared to RN, but viable parenchyma is still often removed, surrounding tissue directly damaged by compression or cautery, and the remaining kidney usually experiences ischemia and likely ischemic damage. ${ }^{1,2}$ The direct surgical injury and nephron loss that occurs during PN or RN is a unique type of insult that places patients at risk for AKI and $\mathrm{CKD}^{3-6}$ (defined as an eGFR $<60 \mathrm{ml} / \mathrm{min} / 1.73 \mathrm{~m}^{2}$ ). eGFR is a calculated estimate of renal function based largely on the $\mathrm{sCr}$ level.

NGAL is a well-studied biomarker for AKI that has been extensively evaluated in adult and pediatric cardiopulmonary bypass patients, ${ }^{7,8}$ kidney transplant patients, ${ }^{9,} 10$ and patients in intensive care settings. ${ }^{11,12} \mathrm{NGAL}$ can detect AKI 24-48 hours before a rise in $\mathrm{sCr}, 7,8,10,12,13$ and the injury detectable by elevated NGAL levels has been shown to better correlate with long-term morbidity and mortality than the limited changes seen in $\mathrm{sCr}$ alone. ${ }^{14}$ This increased sensitivity to injury not detected by $\mathrm{sCr}$ has the potential to identify low-level injury in surgeries like $\mathrm{PN}$, which do not always cause a rise in $\mathrm{sCr}$ to warrant a diagnosis of AKI.

Surgeons often utilize renoprotective techniques to minimize ischemic damage during PN, including kidney cooling with ice slush, administration of intravenous mannitol prior to renal artery clamping, non-ischemic "no-clamp" surgery, and clamping of regional branched arteries to the tumor. . Changes in $\mathrm{sCr}$ after PN are often very small; we chose to control for the possibility of a limited rise in uNGAL levels in PN patients with minimal or no surgically induced AKI by studying RN patients and thoracic surgery patients as control groups. Thoracic surgery patients served as a non-renal control group in which patients had indwelling Foley catheters for 24 hours, limited risk of massive blood loss, and no intraabdominal insufflation to minimize possible surgery-related kidney injury.

The goals of this study were to evaluate uNGAL as a marker for early AKI in patients undergoing PN and to identify the surgical and clinical factors that may be associated with increased intraoperative kidney injury in this group. We evaluated preoperative and postoperative UNGAL levels to evaluate the degree of AKI for patients in the PN, RN, and thoracic surgery groups, as well as reviewed comorbidities and preoperative eGFR and uNGAL levels as predictors of postoperative uNGAL levels.

\section{METHODS}

After institutional review board approval, informed consent was obtained and consecutive renal surgery and thoracic surgery patients of multiple surgeons had urine and serum specimens prospectively collected. PN and RN were performed as previously described ${ }^{15,} 16$ with PN usually performed with ice slush for kidney cooling (cold ischemia) and intravenous mannitol administration. The majority of thoracic surgery patients underwent thoracoscopic surgery with minor to moderate resections. All patients had Foley catheter drainage of the bladder overnight per standard perioperative surgical protocol, which allowed for uniform urine collection practices. 
Urine specimens were collected preoperatively (at the time of bladder catheter placement after induction of general anesthesia), upon arrival to the PACU, and at 4, 8, 12, and 24 hours after completion of the operation. Urine specimens were immediately transported to the clinical laboratory where they were spun, aliquoted, and stored at $-80^{\circ} \mathrm{C}$. The specimens were later thawed and analyzed for NGAL and creatinine levels using a commercially available ELISA assay (R\&D Systems, Minneapolis, MInnesota) that was internally validated in a CLIA-certified clinical laboratory. Serum to measure creatinine was collected as part of our routine preoperative testing and postoperatively on a daily basis during the patients' hospital stay.

We also evaluated the relationship between AKI by AKIN criteria and postoperative uNGAL. AKI is traditionally determined according to AKIN consensus criteria which defines AKI as a $50 \%$ or $0.3 \mathrm{mg} / \mathrm{dl}$ increase in $\mathrm{sCr}$ within 48 hours of insult. ${ }^{17}$ The relationship was assessed using a linear GEE model with an autoregressive correlation structure. Since multiple uNGAL measurements from the same patient are not independent, a GEE model was required to incorporate the correlation between different measures from the same patient. Patients without a preoperative specimen or with fewer than 3 postoperative measurements were excluded from the analyses. An analysis utilizing NGAL cutoffs was performed to confirm the results of the GEE model: $50 \mathrm{ng} / \mathrm{ml}$ or less is normal; greater than $150 \mathrm{ng} / \mathrm{ml}$ is specific for AKI, ${ }^{14}$

GEE models were also used to evaluate postoperative uNGAL and normalized uNGAL (the ratio of urine NGAL to urine creatinine) over time by surgery group, adjusting for baseline marker value. Normalized NGAL was evaluated to correct for possible dilution of UNGAL related to hydration status and the use of intraoperative mannitol. The unadjusted mean biomarker values for each surgery type over time were plotted, with confidence intervals obtained using bootstrapping.

Univariate GEE models were also used to test for associations between UNGAL and baseline clinical features that may be predictive of kidney injury (age at surgery, gender, diabetes, CVD, preoperative UNGAL, normalized UNGAL, creatinine, and eGFR) as well as surgical factors (use and type of ischemia, ischemia duration, estimated blood loss, and mannitol administration), adjusting for preoperative uNGAL. The same analyses were performed for normalized NGAL. Our definition of CVD included the presence of preoperative diabetes, coronary artery disease, heart failure, hypertension, high cholesterol, hyperlipidemia, and high triglycerides.

We evaluated the rates of AKI between patients with preoperative estimated GFR (eGFR) $\checkmark 60 \mathrm{ml} / \mathrm{min} / 1.73 \mathrm{~m}^{2}$ and those $<60 \mathrm{ml} / \mathrm{min} / 1.73 \mathrm{~m}^{2}$ using the chi square test. We also evaluated the effect of preoperative eGFR level on preoperative uNGAL levels using linear regression and postoperative uNGAL levels using a GEE model in patients undergoing partial nephrectomy. The Chronic Kidney Disease Epidemiology Collaboration equation was used to calculate eGFR. ${ }^{18}$ All analyses were conducted using Stata 12.0. 


\section{RESULTS}

Between April 2010 and April 2012, 220 consecutive patients were enrolled in our prospective study. 162 patients (PN: $88, \mathrm{RN}$ : 32; thoracic: 42 ) had adequate specimens to be included in the final analysis. Patient characteristics are shown in table 1. Fewer thoracic surgery patients had diabetes (5\% vs. 13-14\%) than kidney surgery patients, while RN patients had slightly higher rates of cardiovascular disease than thoracic surgery patients, or PN patients (72\%, 67\%, and 65\% respectively). Preoperative uNGAL was highest in the RN group $(10.5 \mathrm{ng} / \mathrm{dl})$ compared to the PN $(5.8 \mathrm{ng} / \mathrm{dl})$, and thoracic surgery groups $(3.3 \mathrm{ng} / \mathrm{dl})$ (Table 1). Conversely, RN patients had the lowest preoperative median eGFR $(61 \mathrm{ml} / \mathrm{min} /$ $\left.1.73 \mathrm{~m}^{2}\right)$ followed by PN patients $\left(78 \mathrm{ml} / \mathrm{min} / 1.73 \mathrm{~m}^{2}\right)$, and thoracic surgery patients $(84.5$ $\mathrm{ml} / \mathrm{min} / 1.73 \mathrm{~m}^{2}$ ).

There was no association between postoperative UNGAL levels and the development of AKI by AKIN criteria within 48 hours. AKI was not significantly associated with elevated uNGAL in patients undergoing PN with an adjusted $6.4 \mathrm{ng} / \mathrm{dl}$ increase in uNGAL for patients with AKI $(\beta=6.4 ; 95 \% \mathrm{CI}-10.5,23.3 ; \mathrm{p}=0.5)$ or $\mathrm{RN}(\beta=-33.43(95 \% \mathrm{CI}-137.45$, 70.58; $\mathrm{p}=0.5$ ). There were a total of 36 patients of $162(22 \%)$ with AKI by AKIN criteria; the median maximum uNGAL level was $39 \mathrm{ng} / \mathrm{ml}$ (IQR 13, 73). Four of these patients had uNGAL levels $>150 \mathrm{ng} / \mathrm{ml}$, one PN, one RN, and two thoracic surgery patients.

Postoperative uNGAL levels were not elevated in the PN group compared to thoracic or RN control groups (Figure 1). Among the three groups, RN patients showed the highest levels of uNGAL after surgery (an average of $43.8 \mathrm{ng} / \mathrm{ml}$ higher than PN; 95\% CI 14.3, 73.3; $\mathrm{p}=0.004$ ). $\mathrm{PN}$ and thoracic patients showed similar uNGAL patterns (difference between groups $2.0 \mathrm{ng} / \mathrm{ml} ; 95 \% \mathrm{CI}-24.9,28.9 ; \mathrm{p}=0.9$ ). Very similar patterns were seen by surgery type when evaluating normalized NGAL. Patients treated with RN had significantly higher normalized NGAL after surgery as compared to PN $(81.88$ (95\% CI 24.83, 138.93; $\mathrm{p}=0.005)$ and thoracic and PN patients exhibited similar levels ( -6.34 (95\% CI $-66.58,53.89 ; \mathrm{p}=0.8)$. Stratification of AKI by uNGAL level $>150 \mathrm{ng} / \mathrm{ml}$ to indicate presence of AKI did not change the relationship between the three groups (data not reported).

Evaluation of baseline features indicative of kidney injury among PN patients (Table 2) revealed that for every 10 -unit $\left(\mathrm{ml} / \mathrm{min} / 1.73 \mathrm{~m}^{2}\right)$ increase in preoperative eGFR, preoperative uNGAL could be expected to decrease by $4 \mathrm{ng} / \mathrm{dl}$ ( $\beta=-4.04 ; 95 \% \mathrm{CI}-7.04,-1.05 ; \mathrm{p}=0.008)$. The use of warm or cold ischemia was associated with an average decrease in postoperative uNGAL of $47 \mathrm{ng} / \mathrm{dl}(\beta=-47.4 ; 95 \% \mathrm{CI}-70.2,-24.6$; $\mathrm{p}<0.0005)$ after adjusting for preoperative uNGAL levels. Normalized NGAL levels, similar to uNGAL levels, were lower in patients with a higher preoperative eGFR $(\beta=-3.48 / 10$ units eGFR; $95 \%$ CI -6.02 , $-0.93 ; \mathrm{p}=0.008)$ and in those who received warm or cold ischemia during PN ( $\beta=-31.0$; $95 \%$ CI $-48.5,-13.5 ; \mathrm{p}=0.001)$. However, the presence of pre-existing CVD was associated with a statistically significant increased postoperative normalized NGAL level ( $\beta=12.7 ; 95 \%$ CI 1.14, 24.2; $\mathrm{p}=0.031)$, but not uNGAL level ( $\beta=11.7 ; 95 \%$ CI $-1.62,24.90 ; \mathrm{p}=0.085)$. Neither the duration of ischemia nor the use of intraoperative mannitol was associated with postoperative uNGAL levels (Table 2). 
Patients in the PN group with a preoperative eGFR $<60 \mathrm{ml} / \mathrm{min} / \mathrm{m}^{2}(\mathrm{n}=18)$ were older (median age 69 years vs. 56 years), had a higher incidence of CVD ( $89 \%$ vs. 59\%), and were more likely to have experienced warm ischemia or no ischemia ( $24 \%$ vs. $16 \%$, and $24 \%$ vs. $3 \%$, respectively) over cold ischemia when compared to patients with a preoperative eGFR $\checkmark 60 \mathrm{ml} / \mathrm{min} / \mathrm{m}^{2}$ (Table 3). In patients with a preoperative eGFR of $<60 \mathrm{ml} / \mathrm{min} / \mathrm{m}^{2}$, there was a significant relationship between preoperative eGFR and preoperative UNGAL, with an average decrease of $0.40 \mathrm{ng} / \mathrm{dl}$ for each $1 \mathrm{ml} / \mathrm{min} / 1.73 \mathrm{~m}^{2}$ increase in eGFR ( $\left.\mathrm{p}=0.017\right)$. This relationship was not evident for eGFR $ð 60 \mathrm{ml} / \mathrm{min} / 1.73 \mathrm{~m}^{2}(\mathrm{p}=0.5)$.

In PN patients with preoperative eGFR $<60 \mathrm{ml} / \mathrm{min} / 1.73 \mathrm{~m}^{2}$, a greater proportion of patients had postoperative AKI compared to patients with an eGFR 260 (33\% vs. 13\%; $\mathrm{p}=0.039$ ). Patients undergoing PN with a preoperative eGFR $<60 \mathrm{ml} / \mathrm{min} / 1.73 \mathrm{~m}^{2}$ also had a higher postoperative rise in uNGAL ( $\beta=33.6$; $95 \%$ CI 14.9, 52.4; $\mathrm{p}<0.0005$; Figure 2$)$ compared to those with a higher eGFR.

\section{DISCUSSION}

We postulated that the ischemic damage during renal artery clamping and the trauma related to kidney tumor resection during PN would result in AKI and hence produce significantly elevated uNGAL levels in these patients at early time points. We did not see the hypothesized results, as there was little difference in postoperative uNGAL levels in PN patients compared to our control group of thoracic surgery patients using either a regression model controlling for preoperative levels or a standardized cutoff of $150 \mathrm{ng} / \mathrm{ml}$. Based on these findings, damage to the kidney during PN using modern surgical and renoprotective techniques appears to be limited and not detectable by uNGAL, even in our relatively large sample of 88 PN patients.

NGAL has been found to correlate with and predict AKI as well as in-hospital morbidity and mortality in patients with global kidney injury after cardiopulmonary bypass surgery, in the setting of sepsis, and after contrast nephropathy. ${ }^{14}$ This, however, is the first clinical study to evaluate UNGAL as a potential marker for AKI in a kidney surgery model compared to non-kidney surgery controls. Furthermore, it is the first study to examine uNGAL levels after unilateral kidney injury unlike previously published studies that only involved patients with global kidney injury and failure.

Whether the lack of elevated uNGAL levels in our study is because minimal damage occurred in the kidney or because the biomarker is not able to acutely determine injury cannot readily be deduced from our data. Modern PN techniques appear to have limited effect on long-term renal function if ischemic time is short (less than 20-25 minutes warm ischemia or cold ischemia). ${ }^{19-21}$ The majority of patients in this study had maximal renal protection during their operation with utilization of cold ischemia and relatively short operative times (median warm and cold ischemia 18 and 29 minutes, respectively). These factors appear to cause little acute renal damage as postoperative uNGAL levels did not rise.

Patients with a preoperative eGFR $<60 \mathrm{ml} / \mathrm{min} / 1.73 \mathrm{~m}^{2}$ did appear to be at higher risk for postoperative AKI by both uNGAL levels and AKIN criteria. These findings support the 
concept that non-modifiable risk factors such as poor preoperative renal function, ${ }^{20}$ and medical comorbidities known to have an adverse effect on kidney function over time, like diabetes and cardiac disease, may be associated with more "brittle" kidneys that are less tolerant of renal artery clamping-induced ischemia.

Furthermore, a preoperative eGFR $<60$ is associated with a higher preoperative uNGAL level. This is consistent with the findings of McIlroy et al. who also found this association in cardiac surgery patients ${ }^{22}$ and Nickolas et. al. who describe an association between increasing NGAL levels and worsening interstitial disease and CKD. ${ }^{23}$ Patients in the current study undergoing PN with a preoperative eGFR $<60 \mathrm{ml} / \mathrm{min} / 1.73 \mathrm{~m}^{2}$ appeared to have a higher postoperative rise in uNGAL ( $\mathrm{p}<0.0005$ ), and a greater proportion of patients had AKI (33\% vs. $13 \%, p=0.039)$ than those patients with an eGFR 260 ; these findings contrast with McIlroy's report that patients with a lower preoperative eGFR appeared to have a blunted rise in $\mathrm{uNGAL}^{22}$

Early postop elevations in UNGAL in the RN group were surprising, though an eventual rise in UNGAL and $\mathrm{sCr}$ were expected. $\mathrm{Yap}^{24}$ did not demonstrate an elevated uNGAL before $24 \mathrm{hrs}$ in their study of donor nephrectomy patients, though the only previous sample was at $5 \mathrm{hrs}$ post op. In that study, as in ours, mean NGAL did not reach AKI levels $(150 \mathrm{ng} / \mathrm{ml})$ even at $24 \mathrm{hrs}$. It is possible that the increased contralateral blood flow associated with nephrectomy ${ }^{25}$ may be responsible for kidney stress and UNGAL release.. The high rate of AKI in the RN group, as calculated by AKIN criteria (which allows for a rise in $\mathrm{sCr}$ within 48 hours after injury), has been seen in up to $75 \%$ of patients in other studies. $^{24}$

Lower uNGAL levels in patients who had warm or cold ischemia compared to no ischemia would seem to suggest a protective effect of kidney ischemia. However, kidney ischemia has been well documented as a source of kidney injury. It is possible that other factors such as squeezing the kidney for hemostasis or greater blood loss associated with a no-clamp PN results in greater kidney injury than a brief period of warm ischemia or a slightly longer period of cold ischemia. Minimal long-term damage to a solitary kidney with warm ischemia under 25 minutes has previously been documented, ${ }^{21}$ and now with these results there appears to be limited AKI with short durations of warm or cold ischemia with an intact contralateral kidney as assessed by uNGAL. A possible confounder is that decreased urine production in the ischemic kidney may mask injury if a high volume of normal urine from the contralateral kidney dilutes NGAL-rich urine from the ischemic kidney. In a porcine bilateral kidney model of unilateral kidney ischemia, the urine output was significantly lower from the ischemic kidney $\left(1 / 25^{\text {th }}\right.$ at 12 hours $){ }^{26}$

\section{CONCLUSION}

uNGAL does not appear to be a useful marker for detection of renal injury in healthy PN patients. However, patients with poorer preoperative renal function have higher baseline uNGAL levels and appear more susceptible to AKI as detected by uNGAL levels and AKIN criteria compared to those with normal eGFR. 


\section{Supplementary Material}

Refer to Web version on PubMed Central for supplementary material.

\section{Abbreviations and Acronyms}

uNGAL Neutrophil gelatinase-associated lipocalin

$\begin{array}{ll}\text { AKI } & \text { acute kidney injury } \\ \text { PN } & \text { partial nephrectomy } \\ \text { RN } & \text { radical nephrectomy } \\ \text { GEE } & \text { generalized estimating equation } \\ \text { eGFR } & \text { estimated glomerular filtration rate } \\ \text { CVD } & \text { Cardiovascular disease } \\ \text { AKIN } & \text { Acute Kidney Injury Network } \\ \text { CKD } & \text { chronic kidney disease } \\ \text { SCr } & \text { serum creatinine } \\ \text { PACU } & \text { post-anesthesia care unit } \\ \text { CLIA } & \text { Clinical Laboratory Improvement Amendments } \\ \text { HAART } & \text { highly active antiretroviral therapy } \\ \text { ICU } & \text { intensive care unit }\end{array}$

\section{REFERENCES}

1. Lane BR, Poggio ED, Herts BR, et al. Renal function assessment in the era of chronic kidney disease: renewed emphasis on renal function centered patient care. J Urol. 2009; 182:435. [PubMed: 19524967]

2. Lane BR, Fergany AF, Weight CJ, et al. Renal functional outcomes after partial nephrectomy with extended ischemic intervals are better than after radical nephrectomy. J Urol. 2010; 184:1286. [PubMed: 20723936]

3. Ishani A, Xue JL, Himmelfarb J, et al. Acute kidney injury increases risk of ESRD among elderly. J Am Soc Nephrol. 2009; 20:223. [PubMed: 19020007]

4. Lassnigg A, Schmidlin D, Mouhieddine M, et al. Minimal changes of serum creatinine predict prognosis in patients after cardiothoracic surgery: a prospective cohort study. J Am Soc Nephrol. 2004; 15:1597. [PubMed: 15153571]

5. Amdur RL, Chawla LS, Amodeo S, et al. Outcomes following diagnosis of acute renal failure in U.S. veterans: focus on acute tubular necrosis. Kidney Int. 2009; 76:1089. [PubMed: 19741590]

6. Ishani A, Nelson D, Clothier B, et al. The magnitude of acute serum creatinine increase after cardiac surgery and the risk of chronic kidney disease, progression of kidney disease, and death. Arch Intern Med. 2011; 171:226. [PubMed: 21325112]

7. Mishra J, Dent C, Tarabishi R, et al. Neutrophil gel(NGAL) as a biomarker for acute renal injury after cardiac surgery. Lancet. 2005; 365:1231. [PubMed: 15811456]

8. Bennett M, Dent CL, Ma Q, et al. Urine NGAL predicts severity of acute kidney injury after cardiac surgery: a prospective study. Clin J Am Soc Nephrol. 2008; 3:665. [PubMed: 18337554]

9. Hall IE, Yarlagadda SG, Coca SG, et al. IL-18 and urinary NGAL predict dialysis and graft recovery after kidney transplantation. J Am Soc Nephrol. 21:189. [PubMed: 19762491] 
10. Parikh CR, Jani A, Mishra J, et al. Urine NGAL and IL-18 are predictive biomarkers for delayed graft function following kidney transplantation. Am J Transplant. 2006; 6:1639. [PubMed: 16827865]

11. Cruz DN, de Cal M, Garzotto F, et al. Plasma neutrophil gelatinase-associated lipocalin is an early biomarker for acute kidney injury in an adult ICU population. Intensive Care Med. 2010; 36:444. [PubMed: 19956925]

12. Endre ZH, Pickering JW, Walker RJ, et al. Improved performance of urinary biomarkers of acute kidney injury in the critically ill by stratification for injury duration and baseline renal function. Kidney Int. 2011; 79:1119. [PubMed: 21307838]

13. Koyner JL, Vaidya VS, Bennett MR, et al. Urinary biomarkers in the clinical prognosis and early detection of acute kidney injury. Clin J Am Soc Nephrol. 2010; 5:2154. [PubMed: 20798258]

14. Haase M, Bellomo R, Devarajan P, et al. Accuracy of neutrophil gelatinaseassociated lipocalin (NGAL) in diagnosis and prognosis in acute kidney injury: a systematic review and meta-analysis. Am J Kidney Dis. 2009; 54:1012. [PubMed: 19850388]

15. Diblasio CJ, Snyder ME, Russo P. Mini-flank supra-11th rib incision for open partial or radical nephrectomy. BJU Int. 2006; 97:149. [PubMed: 16336347]

16. Russo P. Partial nephrectomy for renal cancer (part II): the impact of renal ischaemia, patient preparation, surgical approaches, management of complications and utilization. BJU Int. 2010; 105:1494. [PubMed: 20553456]

17. Mehta RL, Kellum JA, Shah SV, et al. Acute Kidney Injury Network: report of an initiative to improve outcomes in acute kidney injury. Crit Care. 2007; 11:R31. [PubMed: 17331245]

18. Levey AS, Stevens LA, Schmid CH, et al. A new equation to estimate glomerular filtration rate. Ann Intern Med. 2009; 150:604. [PubMed: 19414839]

19. Lane BR, Gill IS, Fergany AF, et al. Limited warm ischemia during elective partial nephrectomy has only a marginal impact on renal functional outcomes. J Urol. 2011; 185:1598. [PubMed: 21419452]

20. Lane BR, Chen H, Morrow M, et al. Increasing use of kidney sparing approaches for localized renal tumors in a community based health system: impact on renal functional outcomes. J Urol. 2011; 186:1229. [PubMed: 21849192]

21. Thompson RH, Lane BR, Lohse CM, et al. Every minute counts when the renal hilum is clamped during partial nephrectomy. Eur Urol. 2010; 58:340. [PubMed: 20825756]

22. McIlroy DR, Wagener G, Lee HT. Neutrophil gelatinase-associated lipocalin and acute kidney injury after cardiac surgery: the effect of baseline renal function on diagnostic performance. Clin J Am Soc Nephrol. 2010; 5:211. [PubMed: 20056755]

23. Nickolas TL, Forster CS, Sise ME, et al. NGAL ( $\operatorname{Lcn} 2)$ monomer is associated with tubulointerstitial damage in chronic kidney disease. Kidney Int. 2012; 82:718. [PubMed: 22695331]

24. Yap S, Park SW, Egan B, et al. Cytokine elevation and transaminitis after laparoscopic donor nephrectomy. Am J Physiol Renal Physiol. 2012; 302:F1104. [PubMed: 22262478]

25. Young LS, Regan MC, Sweeney P, et al. Changes in regional renal blood flow after unilateral nephrectomy using the techniques of autoradiography and microautoradiography. J Urol. 1998; 160:926. [PubMed: 9720589]

26. Silberstein JS, P.C. Su D, Power ND, Tarin TV, Ezell P, Sjoberg DD, Feifer A, Fleisher M, Russo $P$, Touijer KA. Neutrophil gelatinase-associated lipocalin levels in response to unilateral renal ischemia in a novel pilot two-kidney porcine model. British Journal of Urology International. 2013 


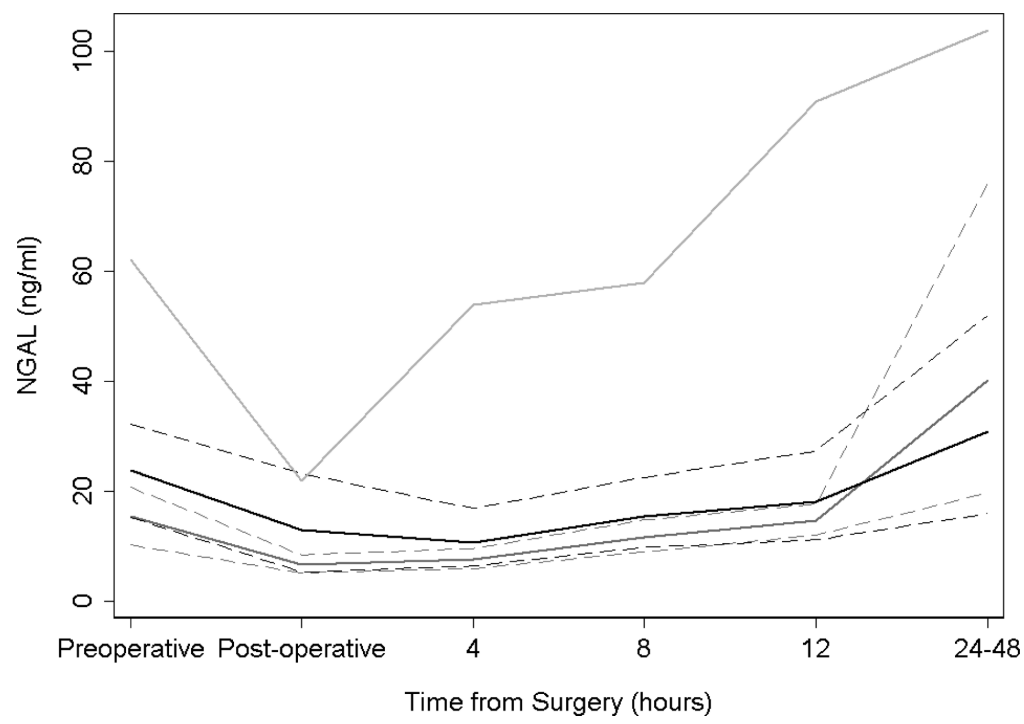

Figure 1.

Urinary neutrophil gelatinase-associated lipocalin $(\mathrm{ng} / \mathrm{ml})$ over time after partial nephrectomy (dark grey), radical nephrectomy (light grey) and thoracic surgery (black) with 95\% confidence intervals (dashed). 


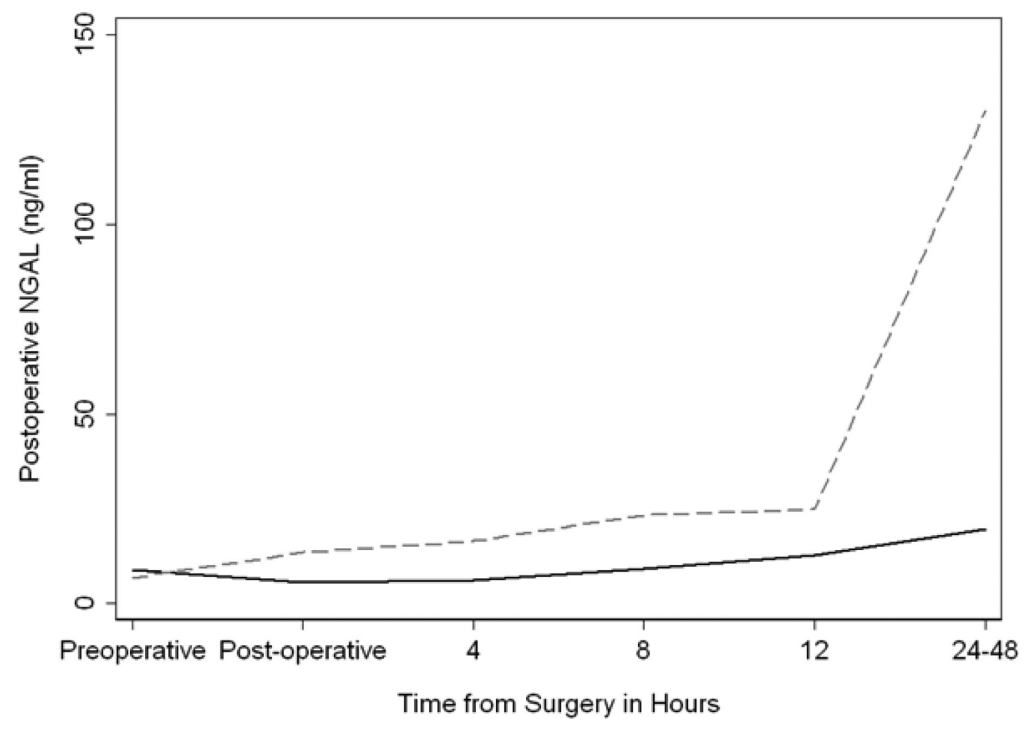

Figure 2.

Urinary neutrophil gelatinase-associated lipocalin (ng/ml) after partial nephrectomy for patients with preoperative estimated glomerular filtration rate scores above $(n=70$; solid) and below ( $\mathrm{n}=18$; dashed) $60 \mathrm{ml} / \mathrm{min}$ per $1.73 \mathrm{~m}^{2}$. 


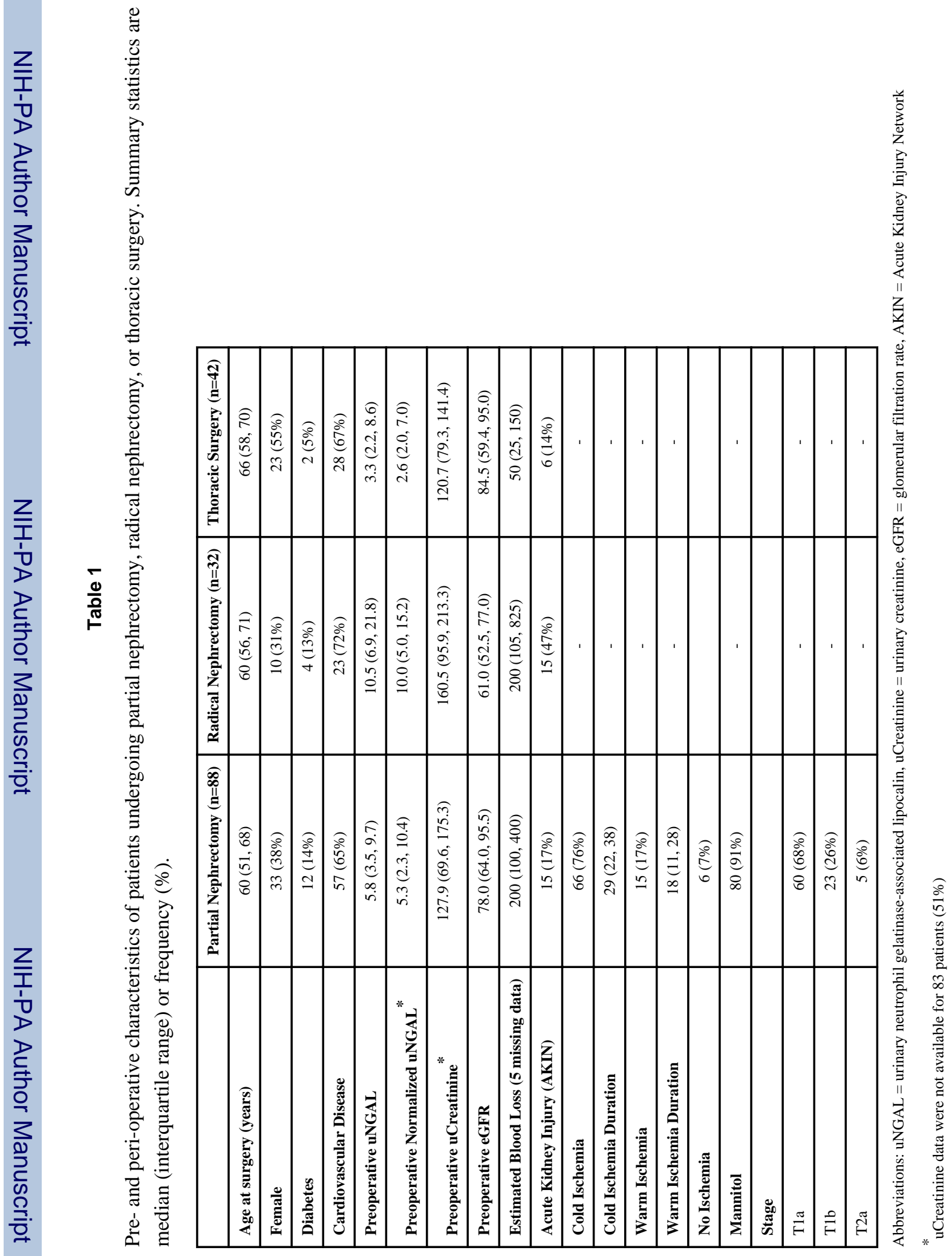




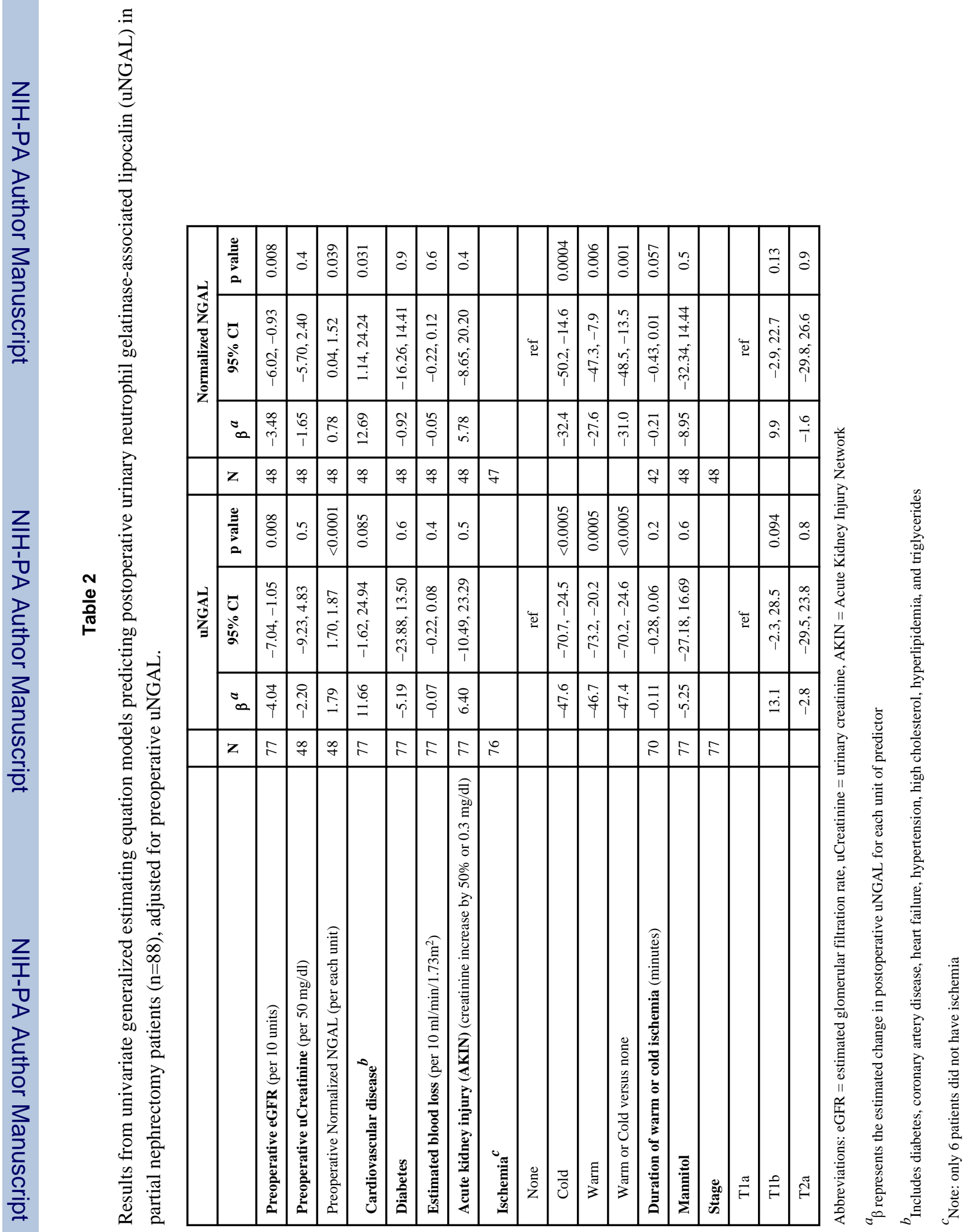

